\title{
Low temperature crystallisation of mesoporous $\mathrm{TiO}_{2}$
}

\author{
Peter Kohn, ${ }^{a}$ Sandeep Pathak, ${ }^{a}$ Morgan Stefik, ${ }^{b \dagger}$ Caterina Ducati, ${ }^{c}$ Ulrich Wiesner, ${ }^{b}$ Ullrich Steiner $^{a}$ \\ and Stefan Guldin $* a \ddagger$
}

\author{
Nanoscale, 2013, 5, 10518-10524 \\ DOI: 10.1039/10.1039/C3NR03009A
}

Received 11th June 2013, Accepted 08th August 2013

First published on the web 12th August 2013

Conducting mesoporous $\mathrm{TiO}_{2}$ is gaining rapidly in importance for the manufacture of green energy applications. To optimise performance, its porosity and crystallinity must be carefully fine-tuned. To this end, we have performed a detailed study on the temperature dependence of $\mathrm{TiO}_{2}$ crystallisation in mesoporous films. Crystal nucleation and growth of initially amorphous $\mathrm{TiO}_{2}$ derived by hydrolytic sol-gel chemistry is compared to the evolution of crystallinity from nanocrystalline building blocks obtained from non-hydrolytic sol-gel chemistry, and mixtures thereof. Our study addresses the question whether the critical temperature for crystal growth can be lowered by the addition of crystalline nucleation seeds.

\section{Introduction}

Mesoporous, nanocrystalline $\mathrm{TiO}_{2}$ is an interesting and useful material for a wide range of applications, such as dyesensitised solar cells ${ }^{1,2}$, photocatalytic reactors ${ }^{3,4}$, electrochemical capacitors $^{5}$, chemical and biochemical sensors 6,7 , and electrochromic devices ${ }^{8}$. These electrochemical devices require an electron-conducting network with pores on the 10 -nm length scale. The nanoarchitecture can be generated by a variety of synthetic routes ${ }^{9}$, among which the sintering of random packed nanoparticles is most widely used ${ }^{10}$. An alternative route with higher morphological control is the structure-directing of inorganic material by supramolecular self-assembly. The driving force for the ordered formation of mesoporous $\mathrm{TiO}_{2}$ on the 5 to $50 \mathrm{~nm}$ length scale is typically the selective association of $\mathrm{TiO}_{2}$ sol via hydrogen bonding with one block of a phase separating block copolymer (BCP). After solution processing the incorporated inorganic network

a Cavendish Laboratory, Department of Physics, University of Cambridge, J. J. Thomson Avenue, Cambridge CB3 OHE, UK.

${ }^{b}$ Department of Materials Science \& Engineering, Cornell University, Ithaca, New York 14853, USA.

${ }^{c}$ Department of Materials Science, University of Cambridge, Trumpington Street, Cambridge CB2 1PZ, UK.

$\dagger$ Present address: Department of Chemistry and Biochemistry, University of South Carolina, Columbia, South Carolina, 29208, USA.

¥ Present address: Department of Materials Science, École Polytechnique Fédérale de Lausanne, 1015 Lausanne, Switzerland. E-mail: Stefan.Guldin@epfl.ch is usually amorphous. A subsequent high temperature step degrades the organic material and crystallises the $\mathrm{TiO}_{2}$ to result in an inorganic nanocrystalline network of high morphological order. This approach has attracted significant interest ${ }^{11}$ because it allows detailed control over pore size ${ }^{12}$ and porosity ${ }^{13}$ of the resulting inorganic thin films.

Using sufficiently high molecular weight BCPs leads to precursor-sol confinement on length-scales that are large enough to allow $\mathrm{TiO}_{2}$ crystallisation at temperatures up to $700^{\circ} \mathrm{C}^{14,15}$. The conversion of the carbon-rich polymer into a sturdy carbonaceous support under an inert atmosphere is a powerful method to further raise the crystallisation temperature to $1000^{\circ} \mathrm{C}^{16}$. Thus, in contrast to the nanoparticle approach $^{10}$, supramolecular co-assembly provides structural stability at extremely high temperatures and therefore leads to the growth of large crystallites and greatly improved charge transport properties ${ }^{15,17}$.

These crystallisation conditions may however not be desirable for a commercial fabrication process. In fact, high processing temperatures are undesired in device fabrication, particularly when using low-cost plastic substrates ${ }^{18}$. Sintering of prefabricated nanoparticles to form an electron-conducting network may be achieved by chemical or mechanical means, yet temperature-induced crystallisation still proves most effective $^{19,20}$. One route to address this challenge is the decoupling of $\mathrm{TiO}_{2}$ crystallisation and photoanode fabrication, e.g. by the transfer of pre-sintered porous films ${ }^{21}$ or the prefabrication of $\mu \mathrm{m}$-sized mesostructured crystalline building blocks $^{22}$. The use of titanate-based precursors has recently been presented as a viable route to crystalline $\mathrm{TiO}_{2}$ networks at low temperatures ${ }^{23}$. Other means of crystallisation that are not temperature-induced include the oriented attachment of nanocrystals ${ }^{24}$ or solution-based growth of $\mathrm{TiO}_{2}$ under non-equilibrium conditions with exposed high-energy $\{001\}$ facets 25,26 .

In contrast to the conventional hydrolytic sol-gel (HSG) chemistry, non-hydrolytic sol-gel (NHSG) synthesis routes allow the formation of $\mathrm{TiO}_{2}$ nanocrystals in solution at quasiroom temperature ${ }^{27,28}$. This has fostered a variety of studies that aim to manufacture mesoporous, nanocrystalline $\mathrm{TiO}_{2}$ at low processing temperatures ${ }^{29,30}$. Hartmann et al. compared the photocatalytic properties of conventional photoan- 
odes that require high temperature crystallisation with photoanodes assembled at low temperature from pre-crystalline building blocks. They found superior performance for the former, which they associated with an improved electronic connectivity within the inorganic network when crystallisation is induced after the morphology built-up ${ }^{29}$.

An appealing strategy to combine both chemical recipes in a "brick and mortar" approach was recently introduced by Bein and coworkers ${ }^{31}$. Here, $\mathrm{TiO}_{2}$ nanocrystals derived from NHSG chemistry are added to a conventional solution of hydrolytic $\mathrm{TiO}_{2}$ sol and structure-directing $\mathrm{BCP}$. The $\mathrm{TiO}_{2}$ nanocrystals ("bricks") disperse in an amorphous $\mathrm{TiO}_{2}$ matrix ("mortar") during the self-assembly process. This combination led to a promising performance when used as photoanodes in dye-sensitised solar cells, with conversion efficiencies of up to $6.0 \%$, for fabrication temperatures as low as $300^{\circ} \mathrm{C}$. They correlated this finding to an enhanced crystallinity of the assembly, induced by the added nanocrystals that act as seeds for the crystallisation of the amorphous material.

In the light of these studies, a further understanding of the crystallisation kinetics in mesoporous $\mathrm{TiO}_{2}$ is of central importance, i.e. it is necessary to understand what are the limiting factors of $\mathrm{TiO}_{2}$ crystal growth at low temperatures. Is this process limited by $\mathrm{TiO}_{2}$ nucleation or growth?

\section{Experimental}

Structure directing agent - In this study a poly(isoprene)-blockpoly(ethylene oxide) (PI- $b$-PEO) BCP with a molecular weight of $M_{n}=34.4 \mathrm{~kg} / \mathrm{mol}$ and a PEO block fraction of $28 \mathrm{wt} \%$ was used. The $\mathrm{BCP}$ was dissolved in an azeotrope solvent mixture of toluene (74.84 $\mathrm{wt} \%)$ and 1-butanol (27.16 wt\%) with a concentration of $10 \mathrm{wt} \%$.

Non-hydrolytic sol-gel (NHSG) crystalline nanoparticle synthesis - Crystalline anatase nanoparticles were synthesised as described in the literature $5,27,29,32$. A dry flask was evacuated and floated with dry nitrogen several times before $5.75 \mathrm{ml}$ absolute ethanol (Fisher Scientific) was added. Then $1 \mathrm{ml}$ of titanium tetrachloride $\left(\mathrm{TiCl}_{4}\right.$, SigmaAldrich, 99.9\% trace metal basis) was injected under continuous stirring. $19.2 \mathrm{ml}$ anhydrous benzyl alcohol (Sigma-Aldrich, 99.8\%) and $0.23 \mathrm{ml}$ of 1,3-propanediol (Aldrich, $98 \%$ ) were subsequently added. The solution was then heated to $T=80^{\circ} \mathrm{C}$ and kept at this temperature for $20 \mathrm{~h}$. $\mathrm{TiO}_{2}$ nanocrystals were precipitated by the addition of $10 \mathrm{ml}$ diethyl ether (Aldrich) to $1 \mathrm{ml}$ of the reaction mixture and subsequent centrifugation for $10 \mathrm{~min}$ at $3750 \mathrm{rpm}$. The wet precipitate was then dried for $1.5 \mathrm{~h}$ at ambient conditions and redissolved in a similar azeotrope solvent mixture, followed by sonication for $2 \mathrm{~h}$. A wide-angle x-ray spectrum of the dried precipitate is shown in the Supporting Information (Fig. S1), demonstrating that anatase crystals were produced during synthesis. A Scherrer analysis of the 101peak width gave nanocrystal sizes between $3.2 \mathrm{~nm}$ and $4.4 \mathrm{~nm}$ for different syntheses. For some experiments the organic ligands were stripped from the nanocrystal surfaces by a phase transfer process involving nitrosonium tetrafluoroborate $\left(\mathrm{NOBF}_{4}\right)$ as described elsewhere $^{33,34}$. As-synthesised nanocrystals were redissolved in hexane $(5 \mathrm{mg} / \mathrm{ml})$ and mixed with an equal volume of $\mathrm{NOBF}_{4}$ in dimethylformamide (DMF, $10 \mathrm{mg} / \mathrm{ml}$ ). The mixture was subsequently stirred until the nanocrystal transfer from the upper hexane to the lower DMF could be visually confirmed. The ligand-stripped nanocrys- tals were then precipitated and washed by the addition of toluene and redispersed in DMF. For the fabrication of mesoporous films, the BCP and the nanoparticle solutions were mixed in a mass ratio of $m_{\mathrm{TiO}_{2}} / m_{\mathrm{BCP}}=1.15$. Films were prepared by drop-casting or spin-coating onto FTO-glass or silicon wafers at ambient conditions. FTO-glass and Si wafers were sonicated in acetone and in isopropanol for $15 \mathrm{~min}$ each before use.

Hydrolytic sol-gel (HSG) synthesis - A titanium-containing sol was prepared by the quick addition of $0.5 \mathrm{ml}$ titanium(IV)isopropoxide (Sigma-Aldrich, 99.999\% trace metal basis) to $0.16 \mathrm{ml}$ hydrochloric acid (37\%, Fluka) under vigorous stirring. Five minutes after addition of the $\mathrm{TiO}_{2}$, precursor the $\mathrm{BCP}$ solution was added in a ratio of $m_{\mathrm{TiO}_{2}} / m_{\mathrm{BCP}}=1.15$, assuming complete conversion of $\mathrm{Ti}$ species to $\mathrm{TiO}_{2}$. Films were prepared by drop-casting or spin-coating onto FTO-glass or silicon wafers at ambient conditions.

HSG-nanocrystal mixtures - In order to investigate the effect of nanocrystalline seeds on $\mathrm{TiO}_{2}$ crystal growth, the NHSG and HSG syntheses were combined. Nanocrystals stemming from the NHSG route were added to the HSG hydrolytic sol in an amount to achieve $m_{\mathrm{TiO}_{2}}(N H S G) / m_{\mathrm{TiO}_{2}}(H S G)=0.15$. For BCP-derived mesoporous samples, a BCP solution was subsequently added to this mixture so that the overall ratio was $m_{\mathrm{TiO}_{2}} / m_{\mathrm{BCP}}=1.15$. Solutions were dropcast onto Si-wafers.

Temperature-induced crystallisation - Unless otherwise stated, the samples were exposed to a temperature protocol consisting of a $10^{\circ} \mathrm{C} / \mathrm{min}$ heat ramp, followed by a $3 \mathrm{~h}$ dwell time at the target temperature and subsequent cooling (Box oven, Cole Parmer EW-3385535). In situ experiments were carried out in an integrated temperature chamber of a diffractometer (Bruker D8 Advance), which was calibrated following the lattice expansion of silicon and $\mathrm{TiO}_{2}$ (rutile).

$X$-ray scattering - X-ray spectra were obtained on a diffractometer with Bragg-Brentano geometry (Bruker D8) and $\mathrm{Cu} \mathrm{K}_{\alpha}$ radiation (step size $0.0182^{\circ}$ ). Correction for the $\mathrm{CuK}_{\alpha} 2$ radiation was achieved by the instruments software (Bruker Eva). The peak of the 101 reflection was subsequently fitted by a Voigt function to relate peak broadening to the finite size of the anatase crystallites. An approximation of Olivero and Longbothum was used to derive the peak width (full width at half maximum) of the Lorentzian and the Gaussian parts ${ }^{35}$. The obtained results were corrected for machine broadening by subtracting (by deconvolution) the broadening of a monocrystalline silicon reference sample. The Debye-Scherrer equation was subsequently employed to obtain a mean crystallite diameter ${ }^{36}$.

Electron Microscopy - Scanning electron microscopy (SEM) was carried on a Leo Ultra 55 with a field emission source at an acceleration voltage of $3 \mathrm{kV}$. For high resolution transmission electron microscopy (HRTEM) a Jeol 4000EX was used with an acceleration voltage of $400 \mathrm{kV}$ and a point-to-point resolution of 0.18 $\mathrm{nm}$. Unit cell parameters for the crystallographic phase identification of anatase $\mathrm{TiO}_{2}$ were taken from ${ }^{37}$, with $a=b=0.373 \mathrm{~nm}$ and $c=0.937 \mathrm{~nm}$ (space group $\mathrm{I}_{1} / \mathrm{amd}$ ).

\section{Results}

Non-templated films - Investigations of films in the absence of a BCP can give valuable information about the underlying processes without the additional complexity of porosity induced by the BCP and the presence of the organic material itself. Figure 1a shows the temperature dependence of 

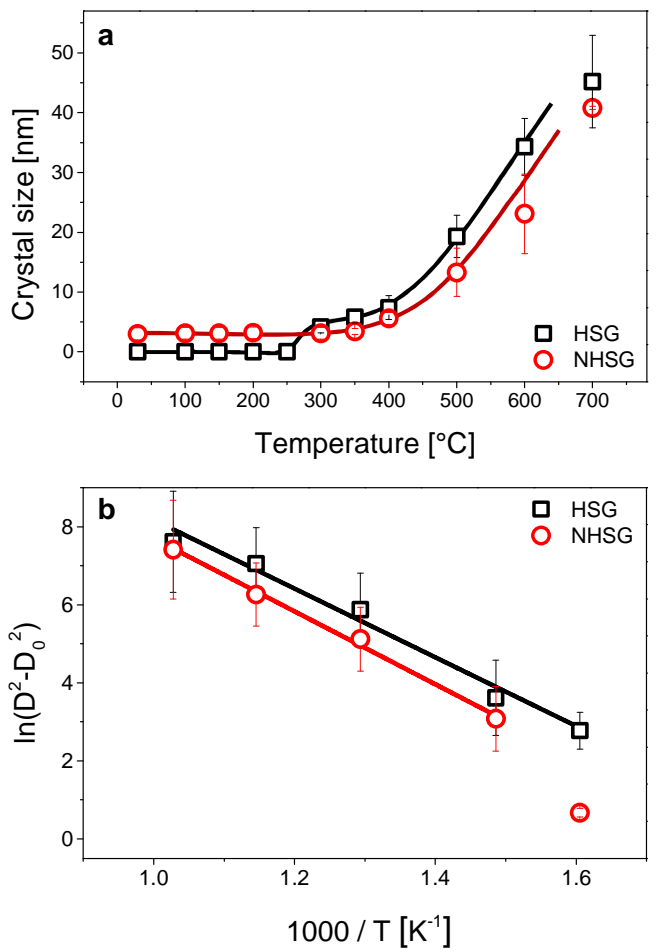

Fig. 1 Temperature-induced crystallisation of non-templated samples: (a) Crystal size as a function of calcination temperature for the HSG (squares) and NHSG (circles) films. Lines are guide to eyes. (b) Determination of the activation energy for crystal grain growth according to Eq. 1.

the mean crystallite size as determined by Scherrer analysis of the $101 \mathrm{x}$-ray reflection. On overview of characteristic XRD pattern is shown in the Supplementary Information. The variability in batch-to-batch results is reflected in the presented error margins. As expected, films prepared by HSG chemistry did not show any crystalline footprint, while a broad peak was observed for films prepared by NHSG chemistry after drying at room temperature (see Fig. SI1 in the Supplementary Information). Both types of films were subsequently exposed to various temperature protocols for temperature-induced crystallisation of the $\mathrm{TiO}_{2}$. To analyse the temperature dependence of the crystal grain size $D$ in more detail, the equation

$$
D^{2}(t, T)=D_{0}^{2}+k \cdot t \cdot \exp \left(-\frac{E_{a}}{R T}\right)
$$

was used, where $D_{0}$ is the initial grain size, $k$ is a temperatureindependent constant, $R$ is the gas constant and $E_{a}$ is the activation energy ${ }^{38}$. All samples have been annealed for a total time of $180 \mathrm{~min}$ at the target temperature. Alongside, we have performed in situ investigations on the kinetics of crystal growth, which is shown in the Supporting Information. Fig. $1 \mathrm{~b}$ and $\mathrm{c}$ show the analysis for HSG and NHSG films, respectively. $D_{0, H S G}=4.2 \mathrm{~nm}$ (first detectable crystal size at $T=300^{\circ} \mathrm{C}$ ) and $D_{0, \mathrm{NHSG}}=3.1 \mathrm{~nm}$ (crystal size after synthesis) yield activation energies of $E_{a, H S G}=73.2 \pm 5.8 \mathrm{~kJ} / \mathrm{mol}$ and $E_{a, N H S G}=76.5 \pm 4.4 \mathrm{~kJ} / \mathrm{mol}$ for the hydrolytic and nonhydrolytic route, respectively. Within experimental errors
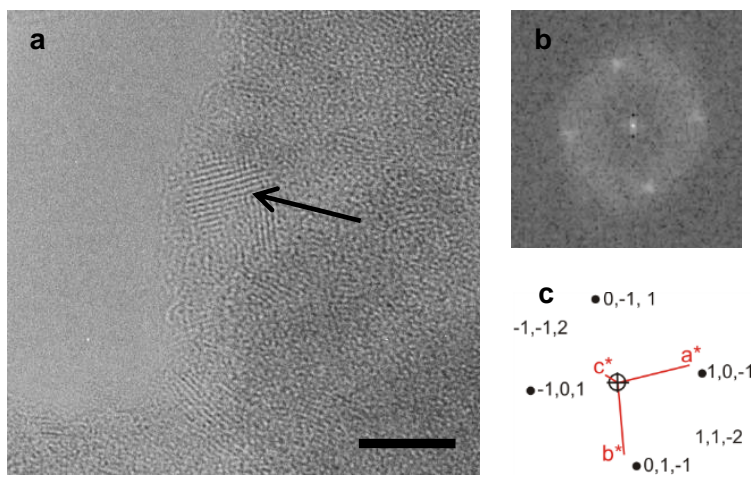

Fig. 2 High resolution transmission electron microscopy of a HSG/NHSG $\mathrm{TiO}_{2}$ mixture. (a) Nanocrystals embedded in the amorphous phase, which completely wets their surfaces. The scale bar represents $5 \mathrm{~nm}$. (b) Fast Fourier Transform and (c) corresponding indexing of the anatase unit cell for the region indicated by the arrow.

both values of $E_{a}$ are comparable, indicating that anatase grain growth may have occurred via the same mechanism in the two synthesis routes. Indeed, Zhang et al. determined an activation energy of $E_{a} \approx 78 \mathrm{~kJ} / \mathrm{mol}$ for anatase recrystallisation by Ostwald ripening ${ }^{39}$, which is close to the values determined from Fig. 1b. We want to emphasise that as in our NHSG route the $\mathrm{TiO}_{2}$ particles were already crystalline after synthesis, i.e. from the beginning of the experiment, the interpretation that the activation energies in Fig. 1b reflect Ostwald ripening of crystalline colloids is highly likely.

In order to identify the role of incorporated seeds on lowtemperature crystallisation of $\mathrm{TiO}_{2}$, amorphous $\mathrm{TiO}_{2}$ synthesised by HSG was mixed with a solution of NHSG-derived nanocrystals in a ratio of $m_{\mathrm{TiO}_{2}}(\mathrm{NHSG}) / m_{\mathrm{TiO}_{2}}(\mathrm{HSG})=0.15$. High resolution transmission electron microscopy (HRTEM) of the fabricated films in Fig. 2 reveals crystalline seeds that are homogeneously dispersed in the amorphous phase ${ }^{40}$. Complete wetting of the nanocrystals by the surrounding amorphous phase walls can be identified. These are two important prerequisites for seed-induced crystal growth. The lattice fringes for the particle in Fig. 2a correspond to the 011 plane of anatase seen along the [111] zone axis, with a spacing of $0.352 \mathrm{~nm}$.

While $\mathrm{TiO}_{2}$ nanocrystal synthesis by NHSG typically involves protection by organic ligands, such as 1,3propanediol $^{29,31,32}$, recent chemical routes allow ligand removal and nanocrystal stabilisation in polar solvents by a $\mathrm{NOBF}_{4}$-mediated phase transfer process ${ }^{33,34}$. Although HRTEM imaging showed complete wetting of the liganddecorated nanocrystals (Fig. 2a), temperature-induced crystallisation was studied for nanocrystals with and without organic ligands.

Both sample types showed weak but pronounced reflection peaks in the diffraction patterns after room temperature synthesis which is consistent with the low volume content of seed crystals. Figure 3a shows the Scherrer crystallite sizes upon annealing. The results of Fig. 1a are indicated as dashed lines for comparison. In contrast to the unseeded HSG, crystal- 

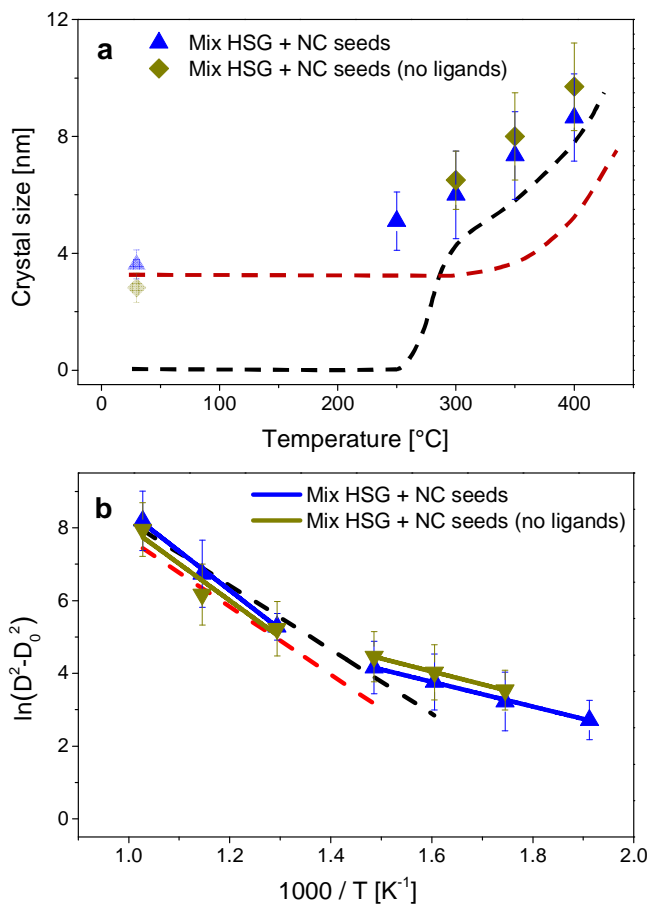

Fig. 3 Effect of $\mathrm{TiO}_{2}$ nanocystal seeds $(15 \mathrm{w} \%)$ on the crystallisation of amorphous $\mathrm{TiO}_{2}$. (a) Crystal size as a function of calcination temperature for mixtures of HSG with ligand-decorated nanocrystals (triangles) and ligand-stripped nanocrystals (diamonds), respectively. (b) Corresponding determination of the activation energy for crystal grain growth according to Eq. 1. Dashed lines indicate the crystallisation behaviour of pure HSG (black) and NHSG (red).

lite growth started at $250{ }^{\circ} \mathrm{C}$ for ligand-covered and stripped nanocrystal seeds. The effect of the seeds is more apparent when calculating the activation energy for crystal growth, plotted in Fig. 3b. In contrast to purely HSG or NHSG samples, two regimes can be observed. Grain growth at temperatures above $400^{\circ} \mathrm{C}$ resembled non-seeded crystallisation with $E_{a}=89.4 \pm 4.2 \mathrm{~kJ} / \mathrm{mol}$ and $E_{a}=84.2 \pm 19.1 \mathrm{~kJ} / \mathrm{mol}$ for ligand-decorated and stripped nanocrystal seeds, respectively. At lower temperatures the experimental data has to be fitted with a lower slope, indicating a different growth mechanism at lower temperatures. The corresponding values of $E_{a}=28.3 \pm 0.1 \mathrm{~kJ} / \mathrm{mol}$ and $E_{a}=29.3 \pm 0.2 \mathrm{~kJ} / \mathrm{mol}$ for the ligand-stripped and ligand-decorated seeds differ by a factor of 2.5 from values obtained from pure HSG and NHSG samples (Fig. 1b). It is important to note that the determination of the activation energy is rather robust towards an uncertainty in the initial seed diameter, yielding from the low crystal content $(15 \mathrm{w} \%)$ in the initial sample before annealing. This is illustrated in the Supplementary Information where we compare $\ln \left(D^{2}-D_{0}^{2}\right)$ for a range of initial grain sizes.

These results are in contrast to previous studies by Zhang et al. who observed similar growth kinetics for both seeded and unseeded samples ${ }^{39}$. This may be due to differing surface chemistries of the nanocrystal seeds, which is decisive for seed-induced growth from a surrounding amorphous phase. While our NHSG nanocrystal seeds exhibit anatase termina-
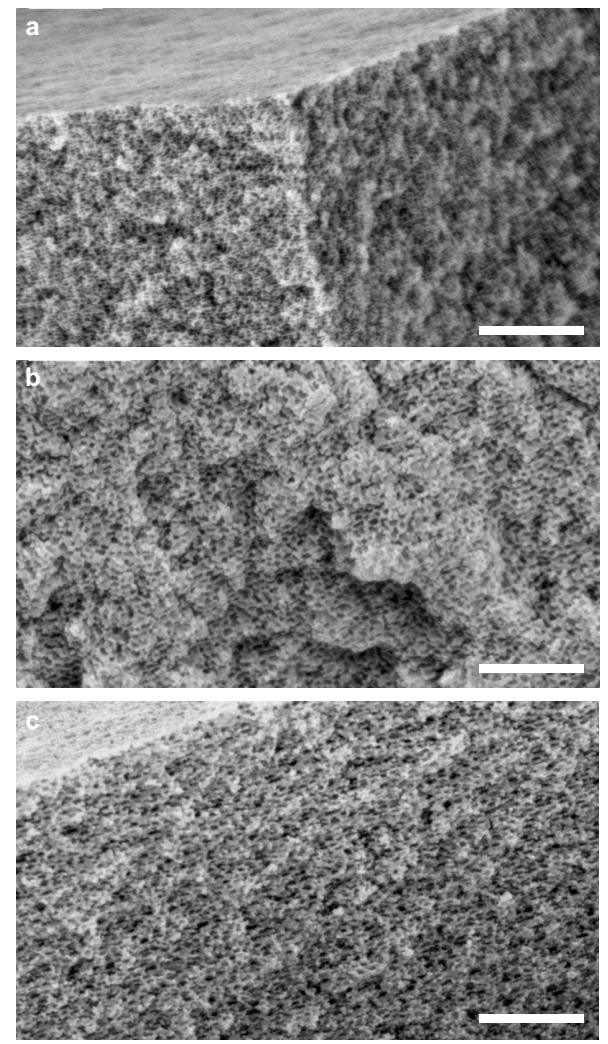

Fig. 4 SEM micrographs of BCP-derived mesoporous films prepared using three different chemical routes under otherwise identical conditions: (a) Pure NHSG (b) pure HSG and (c) HSG with NHSG seeds. The scale bars represent $400 \mathrm{~nm}$.

tion at their edges, as verified by HRTEM, this may be different in the less common $\mathrm{TiO}_{2}$ synthesis of ref ${ }^{39}$. Since the results for ligand-covered and stripped nanocrystal seeds was practically identical, we speculate that the 1,3-propanediol ligands may have already been decomposed at the temperatures for which seed-induced crystal growth was observed .

BCP-derived mesoporous films - The four $\mathrm{TiO}_{2}$ manufacture routes described above were also employed in the synthesis of mesoporous films that use structure-direction by supramolecular co-assembly of the PI- $b$-PEO BCP. In a suitable solvent, PI- $b$-PEO forms micelles with PI cores. As the inorganic components preferentially reside in the PEO phase, the micellar close-packing during solvent evaporation leads to the mesostructuring of the synthesised $\mathrm{TiO}_{2}{ }^{13}$. Figure 4 shows SEM micrographs of fractured cross sections of BCP-derived mesoporous films, stemming from the assembly of (a) NHSG-synthesised crystalline $\mathrm{TiO}_{2}$ nanoparticles, (b) HSG-synthesised amorphous $\mathrm{TiO}_{2}$, and (c) a mixture with a ratio $m_{\mathrm{TiO}_{2}}(\mathrm{NHSG}) / m_{\mathrm{TiO}_{2}}(\mathrm{HSG})=0.15$. The mass ratio of $\mathrm{TiO}_{2}$ to $\mathrm{BCP}$ was kept constant for all samples at $m_{\mathrm{TiO}_{2}} / m_{\mathrm{BCP}}=1.15$. In difference to previously reported results for the Pluronic BCP system ${ }^{31}$, similar mesoporous $\mathrm{TiO}_{2}$ morphologies were obtained after calcination at $T=400^{\circ} \mathrm{C}$ irrespective of the preparation route.

The BCP-derived HSG films crystallised at around $T=$ $350^{\circ} \mathrm{C}$, which is in agreement with Ref ${ }^{5}$. The influence of 


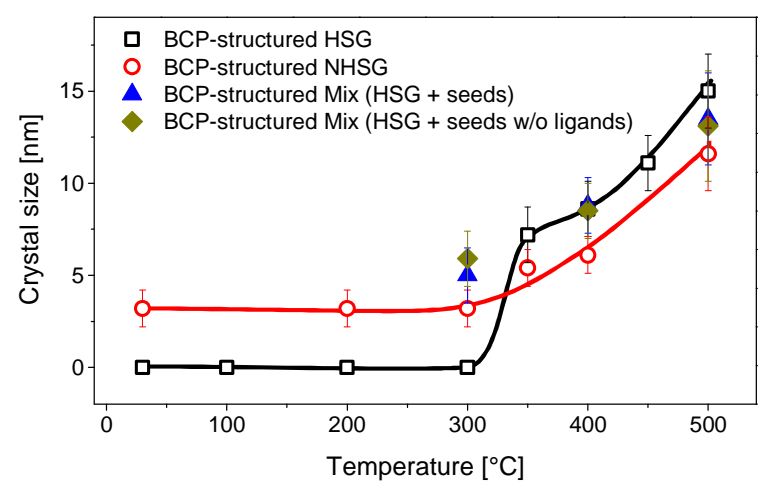

Fig. 5 Effect of selective dissolution of the inorganic component in the PEO phase of the BCP on the mean crystallite size. Mesoporous films were prepared by BCP micellar assembly in HSG, NHSG and seeded HSG/NHSG mixtures, followed by crystallisation at high temperatures.

supramolecular structure formation on crystallisation is evident when comparing Fig. 1 and Fig. 5. Selective dissolution of the amourphous HSG colloids in the PEO phase of the $\mathrm{BCP}$ on the length scale of $10 \mathrm{~nm}$, led to a higher temperature at which $\mathrm{TiO}_{2}$ crystals were first observed. Once the crystal size reached the length scale of the BCP-derived porosity, grain growth was significantly reduced compared to the non-templated samples. This sets in above $500^{\circ} \mathrm{C}$, in agreement with previous studies ${ }^{15}$. The addition of NHSGprepared $\mathrm{TiO}_{2}$ nanocrystals to the $\mathrm{HSG} / \mathrm{BCP}$ mixture resulted in a similar observation as in the non-templated case of Fig. 3: the seeds led to an offset in the critical temperature for crystal growth that can be related to a lowering of the activation energy.

\section{Discussion}

The findings of the experiments described above can be summarised as follows:

1. Initially amorphous $\mathrm{TiO}_{2}$ derived from HSG chemistry and pre-crystalline $\mathrm{TiO}_{2}$ nanoparticles from NHSG chemistry show similar activation energies for temperature-induced grain growth.

2. Both ligand-decorated and ligand-stripped seed crystals embedded in an amorphous $\mathrm{TiO}_{2}$ matrix led to a significant reduction of the activation energy as well as a lowering of the critical temperature for crystal growth by $50^{\circ} \mathrm{C}$.

3. Non-templated $\mathrm{TiO}_{2}$ crystallised at lower temperatures compared to templated films that were structure-directed by BCPs.

4. The grain size at the lowest crystallisation temperature was very similar for templates and non-templated HSG films.

Hydrolytic sol-gel synthesis leads to nanometer-sized, amorphous $\mathrm{TiO}_{2}$-colloids at room temperature, much below the solid-liquid transition temperature of $\mathrm{TiO}_{2}$, i.e. the colloids are glassy. The homogeneous nucleation rate of crystals depends on the volume and the viscosity of the material ${ }^{41-43}$. In macroscopic samples crystallisation mainly occurs by heterogeneous nucleation at impurities which lower the free energy of forming a crystal-amorphous interface. In isolated nanometer-sized objects, homogeneous nucleation can be the dominant mechanism ${ }^{44}$. The fact that NHSG synthesis leads to sub- $5 \mathrm{~nm}$ crystallites, which are stable from ambient conditions to $300^{\circ} \mathrm{C}$, cf. Fig. SI3b, demonstrates that the critical nucleus size in this temperatures well below $5 \mathrm{~nm}$ and thus $\mathrm{TiO}_{2}$ crystallisation is thermodynamically possible in this temperature range.Homogeneous nucleation in a well-defined size distribution of sol particles leads to a characteristic onset time for nucleation, or alternatively for a given heating protocol to a well defined onset temperature, as observed here (i.e. $T=300^{\circ} \mathrm{C}$ for the HSG-derived non-templated samples).

In a fully crystalline system, further grain growth occurs by Ostwald ripening, which is subject to an activation energy that does not depend on the sample history (i.e. is comparable in HSG and NHSG-derived samples, Fig. 1b).

The explanation of the above observations requires a process with two different nucleation rates at the same temperature and which is different for the templates and non-templated case. Observations 1 . and 2 . are readily explained. The nucleation rate in the initially amorphous colloids at low temperatures is slow because of their small size. The amount of crystalline material thus remains low on the time scale of the experiment, leading to the absence of Bragg peaks. With time the amorphous colloids coalesce through Ostwald ripening, increasing their volume. This process is accelerated at increasing temperatures and is hampered in the mesoporous films since there the colloids are surrounded by fewer neighbours, as the colloids are dissolved by the PEO-chain segments and thus diluted.. Once they have reached a certain size the increased nucleation rate plus fast crystallisation of the remaining amorphous material within the colloid leads to appreciable amount of crystallites on the time scale of the experiment. The size of the crystallites then is comparable to the size of the amorphous colloids at this stage. This picture of crystallisation in which homogeneous nucleation is the rate determining step is also supported by other publications ${ }^{45}$.

Since the crystallisation in HSG-produced amorphous $\mathrm{TiO}_{2}$ is dominated by homogeneous nucleation it is reasonable to assume that the crystallisation kinetics can be accelerated by the addition of anatase nanocrystals as seeds. These mixed samples indeed showed substantially reduced activation energies and onset temperatures for crystallisation. The lower onset temperature for otherwise identical experimental conditions is indicative of a lowering of the nucleation barrier, as expected for crystal seeding and in accordance with the above statement of fast crystal growth within the amorphous colloids after homogeneous nucleation has occured. The lowering of the activation energy and the presence of two growth modes in Fig. 3b reflect an additionally observable mechanism for 

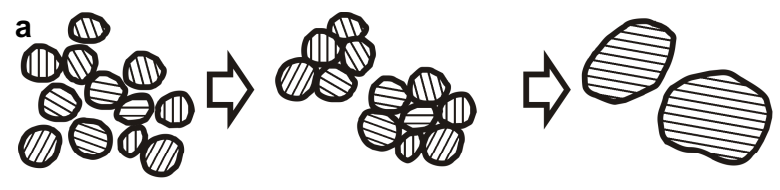

b

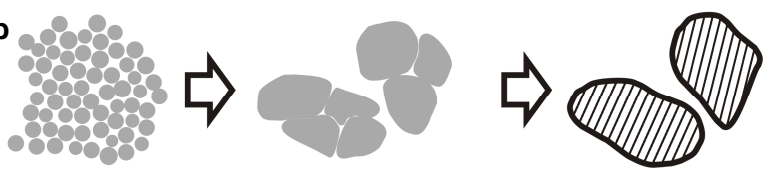

C

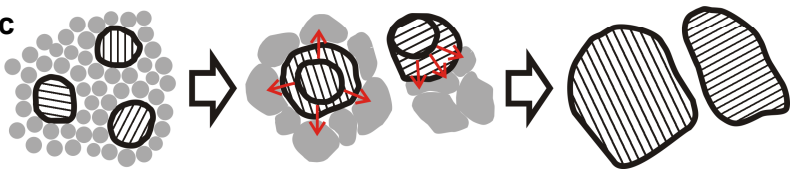

Fig. 6 Rationalisation of observed crystal growth processes for (a) pure NHSG (b) pure HSG and (c) HSG with NHSG seeds (no BCP template).

anatase crystallite growth at low temperatures that is enabled by the presence of crystalline seeds. This mechanism is possibly the growth of the embedded crystallites by diffusion from from the surrounding amorphous phase. The process is replaced by ripening of crystalline colliods at higher temperatures when the samples are fully crystalline, which is identical to the non-seeded HSG and NHSG protocols (Fig. 1b). It is important to note that in the case of mixed $\mathrm{HSG}+$ seeds, we were able to resolve the diffractive footprint of $15 \mathrm{w} \% 2$ $3 \mathrm{~nm}$-sized nanocrystals within the amorphous matrix. This is in contrast to the non-seeded case, where we have not observed any, even weak, crystalline footprint for samples below $T=300^{\circ} \mathrm{C}$.

It is well-known that porosity has an influence on the grain growth ${ }^{38}$ during high temperature sintering: a higher porosity leads to smaller grain sizes at similar temperatures. While solgel-derived ceramics are intrinsically porous on the nanometer length scale, these small pores vanish rapidly at temperatures around $T=350^{\circ} \mathrm{C}$. The larger BCP-derived $10-\mathrm{nm}$ pores are much more temperature stable ${ }^{46}$, as seen in Fig. 4 . The reduced crystal growth kinetics in the mesoporous films is therefore a direct consequence of the local confinement of the crystallites by the pores.

\section{Conclusion}

We have investigated crystal nucleation and growth in mesoporous $\mathrm{TiO}_{2}$. By comparing samples that are purely amorphous (HSG), purely nanocrystalline (NHSG) and mixtures thereof we were able to resolve the parameters that govern crystallisation in mesoporous networks. Our results demonstrate the importance of the colloidal character of sol-gel derived $\mathrm{TiO}_{2}$ particles which may depend on the chosen sol-gel synthesis route. This colloidal nature enables complexation with the PEO polymer segments and therefore allows the material to participate the $\mathrm{BCP}$-driven assembly process. It secondly leads to a crystallisation behaviour that is dominated by heterogeneous nucleation. Nucleation and growth are sub- stantially enhanced by the incorporation of crystalline $\mathrm{TiO}_{2}$ seeds, providing a viable route for the lowering of crystallisation in mesoporous assemblies. Our findings are based on commonly used material platforms and should therefore find widespread implementation for the low temperature fabrication of nanocrystalline electrode architectures, e.g. for dyesensitised solar cells, photocatalytic reactors or electrochemical capacitors.

\section{Acknowledgements}

This project was partially funded by EPSRC and the European Communitys Seventh Framework Programme (FP7/20072013) under grant agreement no. 246124 of the SANS project. $\mathrm{CD}$ acknowledges funding from the ERC under grant number 259619 PHOTO EM. SG is grateful for support by the German Academy of Sciences (Leopoldina).

1 B. O`Regan and M. Grätzel, Nature, 1991, 353, 737-740.

2 A. Hagfeldt, G. Boschloo, L. Sun, L. Kloo and H. Pettersson, Chemical Reviews, 2010, 110, 6595-6663.

3 A. Linsebigler, G. Lu and J. Yates, Chemical Reviews, 1995, 95, 735-758.

4 J. Macak, M. Zlamal, J. Krysa and P. Schmuki, Small, 2007, 3, 300-304.

5 T. Brezesinski, J. Wang, J. Polleux, B. Dunn and S. H. Tolbert, J. Chem. Am. Soc., 2009, 131, 1802-1809.

6 M. Franke, T. Koplin and U. Simon, Small, 2006, 2, 36-50.

7 L. Bonifacio, D. Puzzo, S. Breslav, B. Willey, A. McGeer and G. Ozin, Advanced Materials, 2010, 22, 1351-4.

8 T. Brezesinski, D. Fattakhova-Rohlfing, S. Sallard, M. Antonietti and B. Smarsly, Small, 2006, 2, 1203-1211.

9 X. Chen and S. S. Mao, Chemical Reviews, 2007, 107, 2891-2959.

10 S. Burnside, V. Shklover, C. Barbé, P. Comte, F. Arendse, K. Brooks and M. Grätzel, Chemistry Of Materials, 1998, 10, 2419-2425.

11 C. Sanchez, C. Boissière, D. Grosso, C. Laberty and L. Nicole, Chemistry Of Materials, 2008, 20, 682-737.

12 M. Nedelcu, J. Lee, E. Crossland, S. Warren, M. Orilall, S. Guldin, S. Hüttner, C. Ducati, D. Eder, U. Wiesner, U. Steiner and H. Snaith, Soft Matter, 2009, 5, 134-139.

13 S. Guldin, M. Kolle, M. Stefik, R. Langford, D. Eder, U. Wiesner and U. Steiner, Advanced Materials, 2011, 23, 36643668.

14 D. Fattakhova-Rohlfing, M. Wark, T. Brezesinski, B. M. Smarsly and J. Rathousky, Advanced Functional Materials, 2007, 17, 123-132.

15 S. Guldin, S. Hüttner, P. Tiwana, M. C. Orilall, B. Ülgüt, M. Stefik, P. Docampo, M. Kolle, G. Divitini, C. Ducati, S. A. T. Redfern, H. J. Snaith, U. Wiesner, D. Eder and U. Steiner, Energy Environ. Sci., 4, 2011, 225233.

16 J. Lee, M. Orilall, S. Warren, M. Kamperman, F. DiSalvo and U. Wiesner, Nature Materials, 2008, 7, 222-8.

17 P. Docampo, S. Guldin, U. Steiner and H. J. Snaith, The Journal of Physical Chemistry Letters, 2013, 4, 698-703.

18 B. E. Hardin, H. J. Snaith and M. D. McGehee, Nature Photonics, 2012, 6, 162-169.

19 T. Miyasaka, M. Ikegami and Y. Kijitori, Journal Of The Electrochemical Society, 2007, 154, A455-A461.

20 T. Yamaguchi, N. Tobe, D. Matsumoto, T. Nagai and H. Arakawa, Solar Energy Materials And Solar Cells, 2010, 94, 812-816.

21 M. Durr, A. Schmid, M. Obermaier, S. Rosselli, A. Yasuda and G. Nelles, Nature Materials, 2005, 4, 607-611.

22 M. Nedelcu, S. Guldin, M. Orilall, J. Lee, S. Hüttner, E. Crossland, S. Warren, C. Ducati, P. Laity, D. Eder, U. Wiesner, U. Steiner and H. Snaith, Journal Of Materials Chemistry, 2010, 20, 1261-1268.

23 M. Rawolle, E. V. Braden, M. A. Niedermeier, D. Magerl, 
K. Sarkar, T. Fröschl, N. Hüsing, J. Perlich and P. Müller-Buschbaum, ChemPhysChem, 2012, 13, 2412-2417.

24 N. Tetreault, E. Horvath, T. Moehl, J. Brillet, R. Smajda, S. Bungener, N. Cai, P. Wang, S. M. Zakeeruddin, L. Forro, A. Magrez and M. Graetzel, ACS Nano, 2010, 4, 7644-7650.

25 S. Liu, J. Yu and M. Jaroniec, Chemistry Of Materials, 2011, 23, 40854093.

26 E. Crossland, N. Noel, T. Leijtens, V. Sivaram, J. Alexander-Webber and H. Snaith, Nature, 2013, 495, 215-219.

27 M. Niederberger, M. H. Bartl and G. D. Stucky, Chem. Mater., 2002, 14, 4364-4370.

28 M. Niederberger and G. Garnweitner, Chemistry-A European Journal, 2006, 12, 7282-7302.

29 P. Hartmann, D.-K. Lee, B. M. Smarsly and J. Janek, ACS Nano, 2010, 4, 3147-3154.

30 R. Buonsanti, T. E. Pick, N. Krins, T. J. Richardson, B. A. Helms and D. J. Milliron, Nano Letters, 2012, 12, 3872-3877.

31 J. M. Szeifert, D. Fattakhova-Rohlfing, D. Georgiadou, V. Kalousek, J. Rathousky, D. Kuang, S. Wenger, S. M. Zakeeruddin, M. Grätzel and T. Bein, Chem. Mater, 2009, 21, 1260-1265.

32 M. Niederberger, G. Garnweitner, F. Krumeich, R. Nesper, H. Cölfen and M. Antonietti, Chem. Mater, 2004, 16, 1202-1208.

33 A. Dong, X. Ye, J. Chen, Y. Kang, T. Gordon, J. M. Kikkawa and C. B. Murray, Journal Of The American Chemical Society, 2011, 133, 9981006.

34 I. E. Rauda, R. Buonsanti, L. C. Saldarriaga-Lopez, K. Benjauthrit, L. T. Schelhas, M. Stefik, V. Augustyn, J. Ko, B. Dunn, U. Wiesner, D. J. Milliron and S. H. Tolbert, ACS Nano, 2012, 6, 6386-6399.

35 J. Olivero and R. Longbothum, Journal Of Quantitative Spectroscopy \& Radiative Transfer, 1977, 17, 233-236.

36 P. Scherrer, Nachrichten von der Gesellschaft der Wissenschaften zu Göttingen, math.-physik. Klasse, 1918, 26, 98-100.

37 G. Samsonov, The oxide handbook, IFI/Plenum Press, 1982.

38 H. Gleiter, Acta Mater., 2000, 48, 1-29.

39 H. Zhang and J. F. Banfield, Chem. Mater., 2002, 14, 4145-4154.

40 S. Guldin, P. Kohn, M. Stefik, J. Song, G. Divitini, C. Ducati, U. Wiesner and U. Steiner, Nano Letters, 2013, 13, 5329-5335.

41 D. Turnbull and J. C. Fisher, J. Chem. Phys., 1949, 17, 71-73.

42 H. S. Chen, Rep. Progr. Phys., 1980, 43, 353-432.

43 M. V. Massa and K. Dalnoki-Veress, Phy. Rev. Lett., 2004, 92, 255509.

44 G. Reiter, G. Castelein, A. Röttele and T. Thurn-Albrecht, Phys. Rev. Lett., 2001, 87, 266101.

45 B. L. Kirsch, E. K. Richmann, A. E. Riley and S. H. Tolbert, J. Phys. Chem. B, 2004, 108, 12698-12706.

46 H. Hahn, J. Logas and R. S. Averback, J. Mater. Res., 1990, 5, 609-614. 